\title{
II. MHitteiluthant.
}

\section{Eine japanifhe forftweriammlung.}

Sapan hat nadi bem Mutter ber bentichen Forfingerjammlungen ebenfalls jeine jäbrlichen Bujammentünjte an biverjen forftlich ober fomit

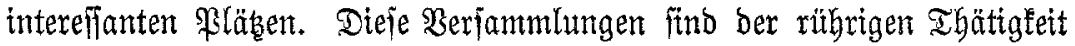
des nahezu zweitaujent Mitglieber umfaffenden Forftvereins zuzujahreiben, Defien Site in Ioftio fich befindet und ber unter ber Jüuhrung eines

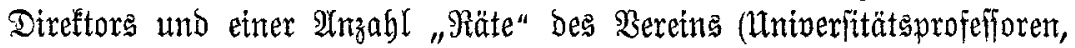
Beamte 2c.) bie veridiebenften $2 \mathfrak{d} f \mathfrak{g a b e n}$ jeiter Beitummung exfült. Dazu gebören in eriter Sinie jogenamnte fleine ßerjammlungen (wemigitents 4 pro $\Im a b r)$ in einem beftimmten Bereinghauje und bie Einleitung unb

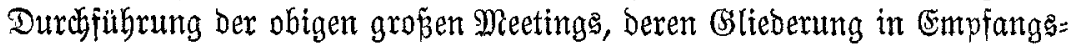
Rebe und Debatte=:ag mit amjhliejender großerer Exturfion und allerlei Feftlichleiten als Begleiterideinung feinerlei Stbmetdung vom fonit im Weften üblichen Programme aufweift. Die Drudelegung einer Monats= Fach= Schrift, eires Dffenen Frage= und 2 Antwort=Brieffaftens für Mit=

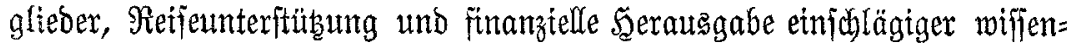

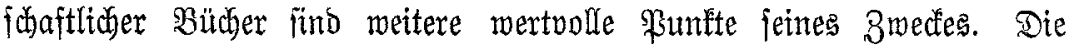
Beiträge Der alleronings meift als "Mitglteder" im meiteften હinne bes Bortes aufzufaffenden "Dereinigten" 2 ngebörigen bes forftlichen uno

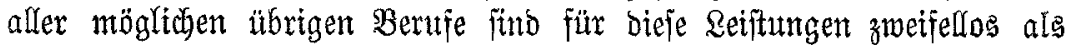

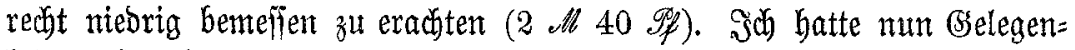

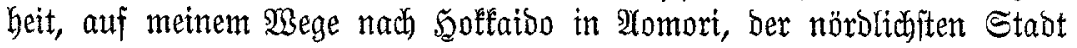
Der Saatintinel, biejex gropen Sabres = Berjammlung beizuwohnen, und ment ich natürlich vorausitichtlich audi nicht ben humbertiten $\mathfrak{I}$ eil bes Gejprodenen zu veritehen boffen fonnte, fo veripract boch bte Reuartigfeit und namentlith die Exturfiton in Den "Atfimape=\$Bald", Den einzigen

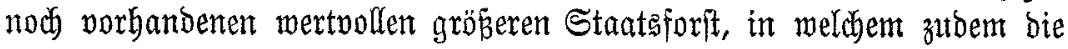
natürlicke Berjüngung ibren Bobnfth aufgeidlagen baben follte, gemug Des Snterelijanten.

Der in ber befaumten einfacten aber gejhmadtollen japanifden Beije deforierte Iurnjanl eimes grof́en Sofulbaules war zum \$ex=

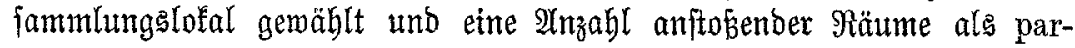
lours benübt, ehe bie cröffnungsceremonie zur grofien Faalle rief.

Sebr mürbig und eindrudfspoll waren bie Eröffmungerede bes \$ereing: Direftors, bie \$egrübung des Bertreters ber ftädtijesen Berwaltung zc. 


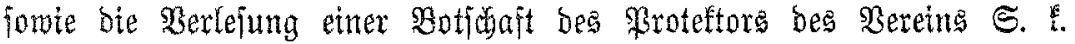
Sobeit bes \$rimen $\mathfrak{F} u$ bimt.

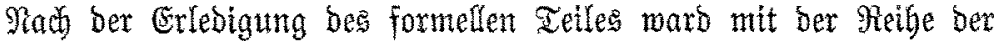
Borträge begonnen und ba bie Rebejallacht einige 20 Rombattanten in Wheficht ftellte, fo modte fie zwei Iage bauern; vorläufig waro fie gegen Pachmittag unterbrochen burch ein Gartenfejt bet einem ber Iempel in ber Mäbe ber Stabt.

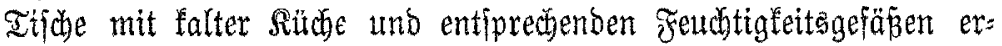
warteten unter freiem Simmel bie Gäite thb neben bem Feuerwer an Gellen Iage war es ingbefonbere bie Farbenpradt ber Gemänber ber bebienenden Resans (Fellnerinnen), weldhe einen bem Europäer unge= wohnten aber angenehmen Einbruaf herborriefen. Retber wurbe bie Freube Durch Regen alsbald getribt.

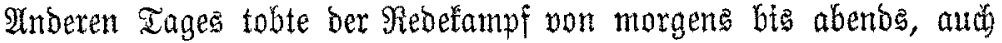

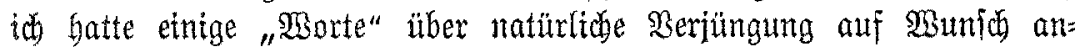
gefügt. (5es befteht hter ein pringipieller Unteridied zwijhen deution und bieitgen Berjanmlungen, indem bier ein befitimmtes Programm mit feitgejebten "Ihematen" nicht aufgeitellt wiro, fonbern jeber "fich Füblenoe" über ein felbit gemäbltes Gebtet jeine Meimung äubert; es

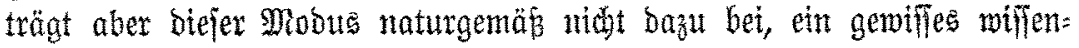
fijaftlides Siveau zu erreichen.

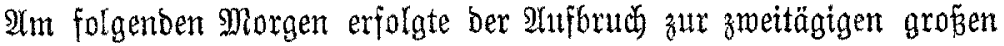

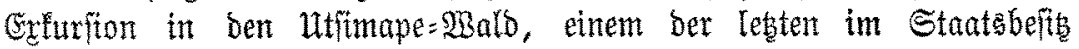
befinbliten wertoollen gröperen fomplere. Das Better war bem

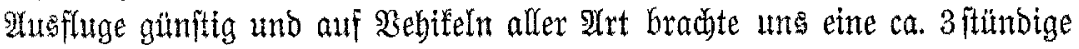

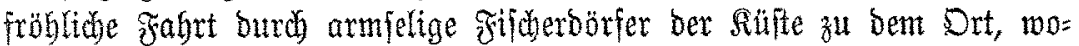
jelbjt bie $\mathfrak{B a g e n}$ verlaffen wurben und bie iebr zablretden Tellnebmer

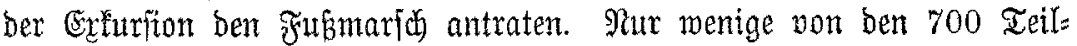

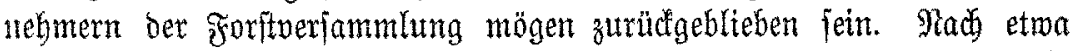

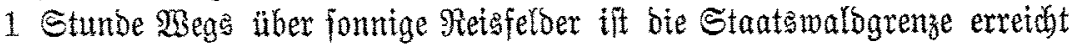
uno in turzer geit fitegt man mitten im 2 alde uno in ber "natürlichen"

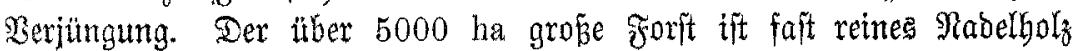
(in ben tieferen Ragen, ebenjo wie in ben bödfften finb etwas blatt= wediflube (Eiden beigemilicht), von Thujopsis dolabrata, japanifo "Ifiunaro", einer ausgejprodienen Sdyattholzart, gebilbet uno itellt in ieinem in Japan fo jeltenen guten Schluffe, feinem atter (ca. 150-200 Эabre) und jeinem bebeutenden Borrate eituen jehr erbebliden Wert bar, obmobl bag Solz ber fibne Schäte bilbenben Thujopsis dolabrata fetteswegs

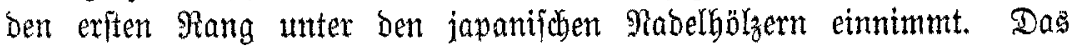
Terrain, weldes von bem warbe bedect it, erweift fid als mittellyobes 
vielgefaltetes Bergland mit ber charafteriftijo vulfanijonen Erjocheinung,

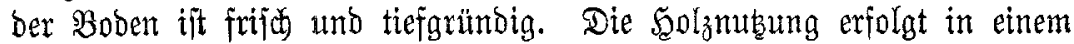
auch beute noch wenig geregelten Plenterbetriebe, ber über ben

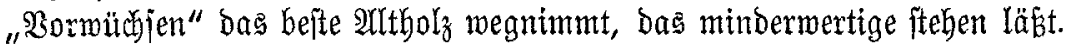

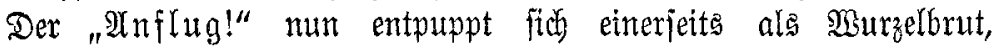

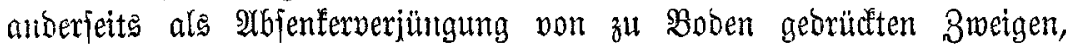
(Eigentümlichfeiten ber Thujopsis dolabrata), jomie auch als Rern=

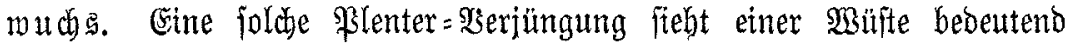
äbnlider als einer "Mirtichaftşbetgätigung", uno menn man bie "Sor=

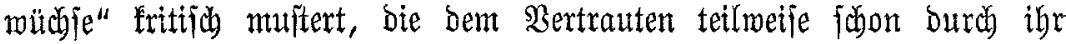

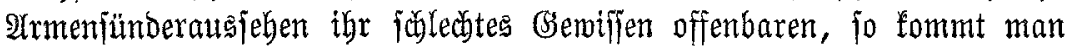
nidjt jelten auf $40,50,60$ ja 70 jäbriges arlter bei $1 \mathrm{~m}$ Şöhe. Eine

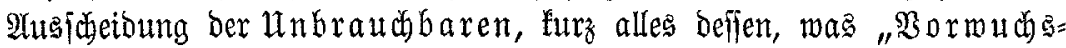
mufterung" und "Siflege" beiff, ift meder befamut, noch beliebt und

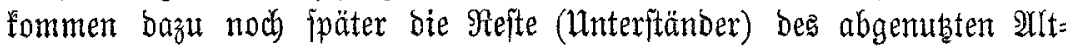
bolzes gropenteils zum Einwachjen, fo ift eine "natürliche Beriüngung", freilith obne Sulturfoften fertig, Ste fich aber zu einer wirtfedaftichen natürlichen Serjüngung etwas fefor biametral entgegengejebt verbält. Wie ubberall in Sapan fo bat bas Feuer auch bier mitunter nambafte Serwültungen angerichtet und find an folchen \$läthen Hinoki (Chamaecyparis obtusa) und Sugi (Cryptomeria japonica) gepflanzt, welde gutes Gebeihen veriprechen. S̈m allgemeinen maren bie Walbbiloer wentg bifferent. Die verjobiedeniten 2 Iltersitufen wechjelten unregelmäbig, überall

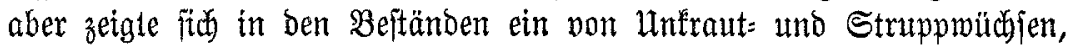
ßambus 2c. freier \$odon, was in Sapan als bödjt jeltener Fall er= fheint und bem gropen Schatten, ben bie bichtgejdlojenen Thujopsis. verbreiten, verbanft wirb.

Gegen Nactimittag mar man an bem "Eamp" für bie Macht an= gefommen. Suftige 3meighütten fanden fich an einem Bache auf einer plateauartigen Erbebung bes uffers aufgejdjlagen und alsbald entfaltete fich ein buntes Treiben und Drängen um die panr Wirtsbudon; man veriorgte fich mit jetnen Ronjerven uno etwas Irinflbarem einitweilen, bis ppäterhin aud Suppe uno bergleichen in ben befaunten Sablzidüüfeln gereicht wurde. Sibgelegenheit (Stuhl, Bant) gab es nitht, fondern man lagerte auf den Matten, welche ben Boben entlang ben $\mathfrak{B a ̈ n d e n ~}$ ber Fultten gelegt waren. Da bie Seitenwände biejer "Molgnungen“

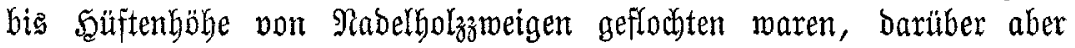
bie balfamijose $\mathfrak{B u f t}$ bis ans Dadb ungehinbert burdftrich, auperbem bie Dadh= Dechung obne lanterlage aud ) nur luftiges (Bezmeige aujwies,

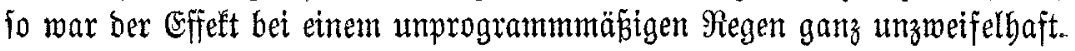




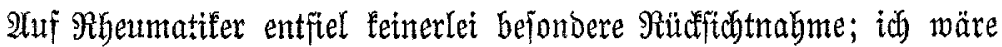
über bie „sobeshymnen" auf bas veranitultende ßomitee, bätte man in Entropa bas verjufft, feinesmegs im 3 meifel gewejen jogar für ben Fall, dás man auci eine relativ warme Sommernadyt, wie fie thatiäblict) eintrat, bätte garantieren fömner. Şier war 2lles froh, munter unb guter Dinge und bas Bild wurbe gegen 2lbent immer eigenartiger; bie Stille

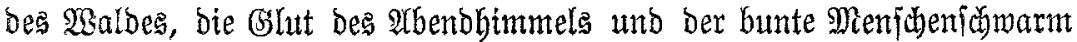
mit ber frembllingenden Spradje - ich zog unwilliutrlich \$arallelen zwijchen ber Begenwart und ber Seimat unb bachte an alles Möglidje, bits man ftch zum Schlafen auf feine Matte ftredte. Olber num fant etne über=

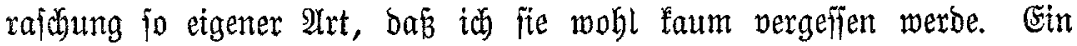

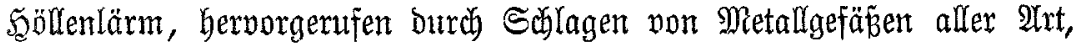

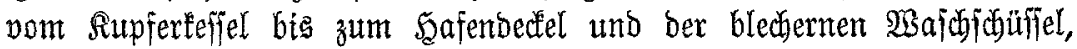
Itellte bie von ein paar Dutend Rutlis gebotente Muflu vor, ber auf bem Futie das papierene plaftifdse Bill bes $\mathfrak{B a l b g o t t e s ~ " D a i t e n g o " ~ f o l g t e , ~}$ innen beleuchtet mit vielen Sierzen uno über huftigem Gerüfte verfertigt, langiam getragen von einer Shar idreiender uno gejtifulterenber 2 Yrbetter (Ruli). Der eingig praftifable saeg fitr diejes Gebilde war ber Bach und bie begreifficten Störungen bes "I Ierrains" verurfachten viel 2 lufent= balt mit "Stimmenaufmand" Der Den Daitengo ichleppenden Menge, bern bas Ganze, io wurbe idh belehrt, war nichts meiter als eine Schautellung, eine Dnation zu Ehren bes bie Gregend mit feinem Bejuche augzeichnenden Forftwereins und veranftaltet von Bewohnern ber Utmgegend, Maldarbettern $2 c$.

Der "Dattengo" ijt fither eine für ung abjonberliche \$erjonififfation

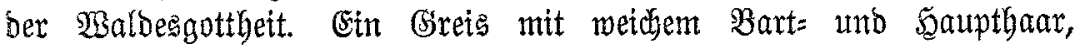

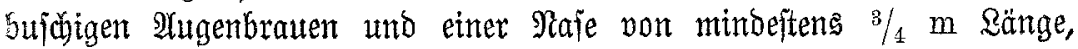
jolange bie normale Rörpergröß̈e eines Mentichen als Unterlage ber \$roportion ber Darittellung in Frage fommt; bie linte F̧and bält ein Blatt unb bie ganze Figur ift in ein blutrotes Bewand gefleidet. Man

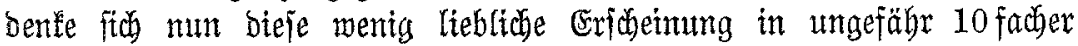
sebengrö̈Be, von Papier gemacht, imnen beleudutet und burd bie vom

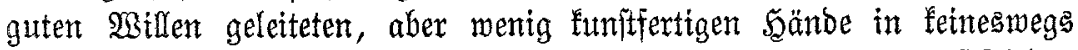

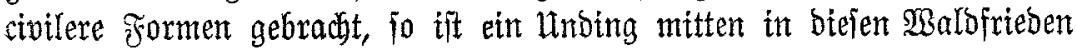
geitellt, für bas bem Europäer bas Berftänonis fehlt. Unjer Daitengo batte eine minbeitens $1 \frac{1}{2} \mathrm{~m}$ lange rote, Murft" $^{\text {"Naje }}$ und eine unglaubliche "Mitene".

Das Bergnügen jobien jeinen Şöbepuntt zat erreidjen, als bie Be=

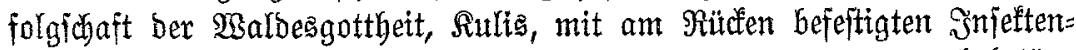
flïgelimitationen aus \$apier burch alle Şütten tanżten unter obrbetäu= 


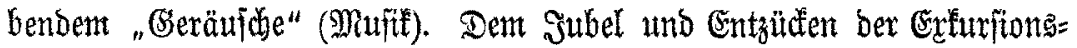
teilnebmer nach hatte bie Sache etnen burdjichlagenden Errolg.

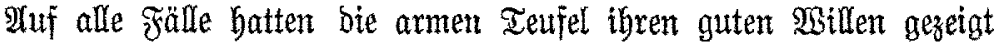
unb eine Fleine Sammlung in usum ..... ward burd entiprechente Irfobolbegeifterung alsbald fidyt= und börbar von ben Berantaltern quittiert.

Dex näd)fte Morgen führte bie Errfurfion nad̆ mehritündigem Maridie, ber befondere Beridjiebenheiten Des Forites mur infofern auf: wies, als einige mit Eichen gemijidte Particen pajfitert wurben, enblid gegen Nachmittag nadh einem auberbalb bes $\mathfrak{B a l b e s}$ gelegenen, multer= baften Forftgarten. Dort mutrbe ber Shlü Der Serjammlung in cere=

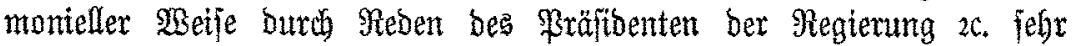
feterlid) voffzogen uno gegen 2 beno von ber benadbarten (Eijenbahnitation

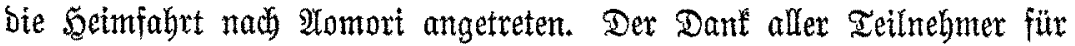

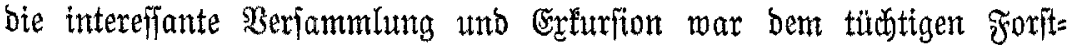
verein fither und fo jet ihm aud von mir etn "Floreat, crescat" von Sergen gemünjigt.

Iolto.

Dr. Şẹ̃ele.

\section{Derjammlung des forftwereins für Eljaḱ:

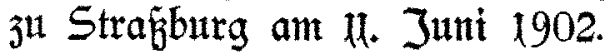

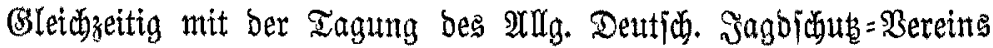

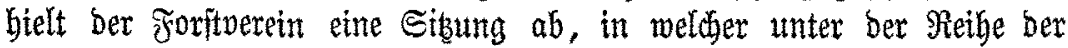
Bereinsangelegentheiten bejdloffen wutroe, im näbjten Sabre mieber einen Musflug in bie Foriten eines Mabjbaritaates zu unternehmen utro bei biejer Belegenheit an pafjendem Drte bas Ihema za bebandeln:

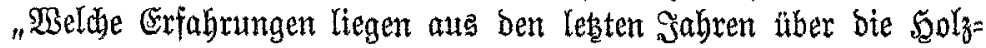
verwertung vor in Begtig

a) auf bie Mrt Der Mertäufe? (Submiffions=, öffentlider, $\mathfrak{B o r}=$ Rach=, Freithand:\$erteuf.)

b) auf bie Rogatnteilung?

c) auf bie Berwertung ber Mubbölzer in Bemeindewalbungen?"

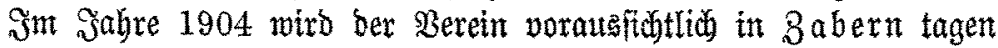
und von ba einen $\mathfrak{A}$ thffug in bie 1897 von Sturm tmb Sagel arg beint=

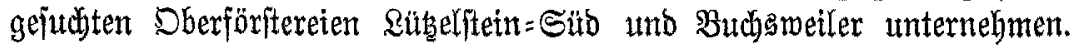

Sterauf wurbe als biesłähriger hauptberatungeggegenftanto bie beut=

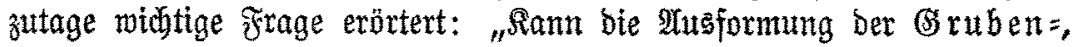

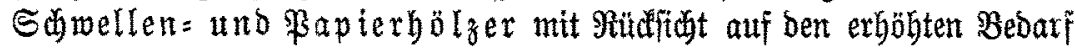

